tonics, perhaps even as tissue-builders, being overlooked. As such they will certainly be found of great value in certain stages of treatment in appropriate cases. Indeed, this their legitimate use is very largely and increasingly recognised by the profession, and they are, with the lactophosphates of lime and iron, perhaps the best general tonics both in cases of incipient consumption and in the more advanced stages of that disease, when the progress of the disease has been arrested.

Ventnor, I.W.

\section{NOTES OF A CASE OF HYDROPHOBIA, IN WHICH THE HOT-AIR BATH WAS EMPLOYED.}

By F. A. SOUTHAM, M.A. OXON., F.R.C.S., RESIDENT SURGICAL OFFICER, MANCHESTER ROYAL INFIRMARY.

Susan $\mathrm{T}-$, aged twenty-eight years, unmarried, a strong, well-developed servant-girl, was bitten on March 4 th, 1879, by a large mastiff dog, which was immediately afterwards destroyed, thus preventing any chance of definitely ascertaining the presence of rabies, though this was not at the time suspected. The wound, which was inflicted on the left wrist, was not cauterised, and readily healed. On April 11th, nearly six weeks after the injury, having in the meantime enjoyed good health and apparently forgotten all about the bite, she began to complain of pain in the cicatrix of the wound and up the left arm, of feeling chilly, and also of an aching sensation in the back and limbs. On the following day she was somewhat better, but on April 13th she manifested symptoms of great aversion to fluids, and complained of a sensation of suffocation and difficulty in breathing. The next day, April 14th, there was almost complete inability to swallow, and this, along with the other symptoms which were present, clearly indicating that the patient was suffering from hydrophobia, she was removed the same evening to the hospital.

Condition on admission at 9 P.M.-Perfectly conscions excited and anxious expression ; begged that no one would approach her as she felt so nervous. Able to walk without assistance Aerophobia very well marked, the slightest draught or breath of air bringing on a paroxysm. Extreme dread of fluids, shuddering at the mention of water, and begging us not to bring it near her. Complains at once of the draught, and starts up in bed if the door is opened or left ajar. Respiration 32, irregular, short, and gasping, interrupted from time to time by a deep sigh; pulse 136, very weak; temperature $98.8^{\circ}$; skin slightly moist; pupils dilated, conjunctivæ somewhat suffused. Complains of difficulty of breathing and feeling of suffocation, with sensation of tightness over diaphragm. Says she has now no pain in the old cicatrix, and begs us not to remind her of the wound. Constantly expectorates viscid frothy saliva. Suffers from constant spasms, affecting chiefly the muscles of respiration, causing her to start up in bed and gasp for breath, especially if anyone approaches her. An attempt to get her to swallow some milk brought on a very severe paroxysm.-9.30 P.M.: One-sixth of a grain of curara in a freshly prepared solution was injected into the arm, and as this gave no relief to the symptoms, half a grain of morphia was injected subcutaneously half an hour afterwards. 11 P.M.: The condition of the patient had become much worse. The breathing was extremely embarrassed, having become very quick, shallow, gasping, and more irregular The countenance was dusky, and the lips of a livid hue. The paroxysms became more frequent and severe. She complained of great difficulty in breathing, with a sense of intense suffocation and inability to clear her throat of mucus. She kept continually expectorating tenacious saliva, and the rattling of the mucus in her throat could be heard at some distance. She remained perfectly conscious, talking rationally in a low whisper.

Thinking that the increased difficulty in breathing and signs of deficient aeration of the blood might be perhaps due to the physiological action of the curara, it was thought advisable to discontinue its administration, and, after consultation with Dr. Graham Steell, resident medical officer, I determined to try the effect of a hot-air bath upon the patient. A rough kind of Turkish bath was therefore improvised. The patient was wrapped in blankets up to the neck, a bed-cage covered with blankets was placed over her body and legs, and a large spirit-lamp placed beneath the cage at the bottom of the bed. The necessary preparations for carrying out this treatment excited such severe spasms that we were almost obliged to desist; but as the patient submitted quite willingly, and tried to assist us in every way, being anxious to obtain some relief, we managed to accomplish our object, and at 11.45 P.M. the apparatus was working, and the body of the patient, as she lay in bed, surrounded with an atmosphere of hot dry air. In less than a quarter of an hour a marked change was observed in her condition. The spasms became gradually less frequent and less severe, and in the course of another half-hour had alto. gether disappeared; the breathing at the same time gradually improved, becoming more regular, deeper, and not so rapid. She said that the warmth gave her a feeling of great relief, and her improved colour and general appearance betokened a change for the better. This treatment was continued for an hour and a half, the patient in the meantime perspiring freely, and at 1.15 A.M. the lamp was removed, the body wrapped in blankets, and hot bottles placed on either side. 1.30 A.M. : Temperature $988^{\circ}$; pulse 130 ; respiration 32 . The breathing had now become quiet and regular, and the paroxysms, with the exception of an occasional slight catch in the breathing, had entirely ceased. The aerophobia had quite disappeared, the countenance was less dusky, and she lay quietly in bed, feeling perfectly easy, and expressing a desire to go to sleep. The discharge of saliva from the mouth had stopped, and she experienced no pain nor discomfort with the exception of a feeling of rawness about the sternum. No mention of fluids nor attempt to give her any food was made for fear of exciting a return of the spasms. She still remained perfectly conscious and rational, expressing her gratitude for the relief we had afforded her by means of the hot-air bath.

After this there was no change in the symptoms, the patient remaining quite easy and comfortable, dropping of to sleep for fifteen or twenty minutes at a time, and waking up without any spasm or recurrence of her former symptoms. At 3.30 A.M., after having enjoyed a calm sleep of about half an hour, she all at once awoke with a violent paroxysm, starting up in bed with a copious discharge of saliva from the mouth, and died very suddenly, apparently from spasm of the glottis. The urine had a specific gravity of 1030 , acid; slight mucous deposit; large quantity of albumen and sugar present.

The post-mortem examination showed, as usual, no morbid changes, all the organs appearing to the naked eye perfectly healthy, with the exception of a little undue vascularisation about the pons and medulla, especially in the neighbourhood of the fourth ventricle.

Remarks. - This is the fifth case of hydrophobia which has been treated in this hospital during my residence here in the course of the last twelve months. The condition of the urine in hydrophobia is interesting, for in all five cases it was loaded with albumen; while in this, as also in two of the other cases, sugar was present in considerable quantity. The glycosuria cannot be attributed to the physiological action of the curara, for this condition was present in two cases in which this drug was not exhibited.

As regards the temperature of the patient, it appears to vary very considerably. In two of the four cases to which I have referred it was found to rise slowly and steadily, the temperature in the axilla shortly before death registering in the respective cases $1004^{\circ}, 103 \cdot 2^{\circ}, 101 \cdot 4^{\circ}$, and $105^{\circ}$. In the present instance tine absence of any rise may have perhaps been due to the free diaphoresis which was excited by the hot-air bath.

Of the four other cases, all of which proved fatal, one was treated with subcutaneous injections of chloral hydrate and morphia, one with chloral alone, one with curara and morphia, and one with morphia alone.

With regard to the employment of chloral and morphia, these drugs appear to have very little influence over the course of the disease, merely masking the symptoms by narcotising the patient, and before this object can be attained they have to be administered in large and almost poisonous doses. Curara is, I think, a very dangerous remedy, as in the previous case alarming symptoms of failure of the respiratory power suddenly appeared after two injections of one-quarter grain each at an interval of three 
hours had been given. In the present instance the marked embarrassment of the breathing, as shown by its irregular, rapid, and shallow character, together with the cyanotic appearances of the patient shortly after one-sixth of a grain had been injected, may, I think, have been very probably due to the toxic action of the drug.

The employment of hot-air and vapour baths, old and popular remedies in the treatment of hydrophobia, with the view of eliminating the poison from the blood by means of the free diaphoresis which is thereby induced, appears to be a not altogether irrational plan of treatment, and is, I think, worthy of further trial in the absence of any known antidote for the poison. The marked relief to the patient and alleviation in the symptoms which it at once afforded in the present instance, and the failure of the drugs which I have mentioned in the other cases, would, if another case presented itself, lead me to at once have recourse to this plan of treatment, and I have published these notes in the hope that others may be induced to give it a similar trial.

\section{OBSERVATIONS ON CATARACT IN INDIA;} WITH AN ANALYSIS OF 512 CASES OPERATED ON IN THE AZAMGARH HOSPITAL DURING THE YEAR 1878.

BY P. J. FREYER, A.B., M.D., SURGEON H.M. BENGAL ARMY ; OFFICIATING CIVIL SURGEON, N.W. PROVINCES.

(Concluded from p. 314.)

AMONGST the 512 cases of cataract which are the immediate subject of this paper, the congenital variety occurred only once. It was instanced in a female child aged seven years, who had been blind from her birth. She appeared to have no perception of light, but still I thought it right to try the effect of an operation on one eye. For reasons that will appear hereafter, extraction of the lens was the operation performed. Though the procedure was attended by no mishap, and the wound healed naturally, no vision resulted. Ophthalmoscopic examination of the eye revealed atrophy of the optic disc.

There was only one instance of traumatic cataract, which cccurred in a man aged seventy years. The disease resulted from a blow on the eye by a stick. The lens was extracted successfully.

There were eight cases of soft cataract. The average age in these cases at the time of operation was thirty-nine years, but in all of them the opacity of the lens had existed several years.

In 494 instances the cataracts were of either the mixed or hard varieties; but in many of these, owing to the length of time the disease had existed, it was impossible to say whether the degeneration had commenced in the cortical or nuclear portion of the lens. The average age of the persons amongst whom these 494 cases of cataract occurred was approximately fifty-five years. It must be remembered, however, that in the large majority of them the cataracts had existed mature for several years previous to the time they came under observation; so that the average age at which cataract occurred amongst these people was considerably below this figure.

Amongst the cases mentioned in last paragraph there were several instances of the variety known as Morgagnian cataract, in which a small hard nucleus is surrounded by, and moves about in, a thin milky fluid.

There were two cases of black cataract, in which the lenses were uniformly dense and dark throughout their whole extent. They resembled each other in the fact that in both cases there was complete absence of even sensation of light. In one case I extracted the lens with success, good vision resulting. In the other instance, though the operation was attended by no accident and the wound healed normally, the patient obtained no vision. There had been no history of glaucoma, the eye was healthy-looking in every respect after the operation, and the ophthalmoscope revealed no abnormality in the deep-seated structures, but there was no vision, not even perception of light. I did not operate on the second eye, which presented a similar appearance.
There were two instances of bony cataract, in which the lens had undergone calcareous degeneration. The appearance of the lens before operation was that of a piece of polished ivory, and after extraction it was extremely dense and hard. In each case only one eye was thus affected, the other eye suffering from ordinary hard cataract. In both cases extraction of the lens was performed successfully.

Three instances of capsular cataract occurred. In each of these the cataract consisted merely of an opaque capsule obscuring vision, the lenticular portion having been apparently absorbed. In two of these cases I extracted the opaque capsule by means of iris forceps introduced through a small linear incision in the cornea close to the sclerocorneal junction. In both instances good vision resulted from the operation. In the remaining case I lacerated the capsule by means of two needles introduced through the cornea at opposite points. In this case the result was unsatisfactory, the patient leaving the hospital with scarcely any vision in the eye operated on, and refusing to submit to a second operation.

Three cases occurred in which, the central portion of the lens being opaque and the edges translucent, there were circumstances that rendered extraction of the lens in. advisable. In each of these I made an artificial pupil by iridectomy opposite the translucent edge. In all three cases the operation was successful, having been followed by good vision in each instance.

In the remaining 506 cases the operation undertaken for the restoration of vision was extraction of the lens. The operation performed in all instances was the modified von Graefe's linear extraction after the manner described by Mr. Brudenell Carter in his work on Diseases of the Eye, with this difference, however, that in most cases the incision was made wholly through the cornea, but as close to the sclerocorneal junction as possible. From practical experience, I am inclined to think that better results will be obtained, in India at least, by making the incision wholly through the cornea than wholly or partially through the sclerotic; but the incision should be extremely close to (almost in) the sclero-corneal junction. I am still in the habit of making the incision through the sclerotic where the anterior chamber of the eye is very deep and the vicinity of the ciliary body can be easily avoided; but my experience is that, as a general rule, when the incision is made through the sclerotic there is a tendency to iritis supervening. In some instances I have found it convenient to modify the direction of the corneal incision, owing to opacity of the cornea or peculiarities in the general conformation of the eyeball existing. An iridectomy was performed in all cases before rupturing the capsule of the lens, except in a few instances in which the pupil was very widely dilated, and the lens in its capsule slipped out after making the corneal incision, almost by the mere tension of the vitreous aided by the faintest external pressure.

The patient was anæsthetised in every instance during the operation, the anæsthetic used being chloroform. I consider that too much stress cannot be laid on the absolute necessity of seeing that anæesthesia is thorough and complete before commencing the operation. Not alone is it essential that sensation should be completely in abeyance, but the muscles of the eye should be perfectly passive and incapable of contraction under reflex action. I know of no other operation in which a profound anæesthesia is more absolutely essential than in extraction of the lens. It is very necessary to guard against being deceived by that apparent insensibility that occasionally occurs during the administration of chloroform just before the struggling stage sets in. The quantity of chloroform inhaled will be the best guide against falling into such a mistake; for whilst this seeming insensibility usually takes place when somewhat less than a drachm of chloroform has been used, I find that, owing to the extreme volatility of the drug in India, it generally requires not less than three drachms to effect complete anæesthesia.

Of the 506 operations for extraction of the lens 342 were successful, good vision resulting; 40 were partially successful, fair vision resulting; 111 were unsuccessful, in which little or no vision resulted; and in 13 instances the results are unknown, the patients having left hospital without leave after the operation. Thus, of the total number 0 operations, in 75.5 per cent. good or fair vision was obtained; and in 24.5 per cent. the result was either unsucf ceseful or unknown. I may, however, mention that in a few of the cases returned as unsuccessful, and in which the 